\title{
Low dose unfractionated heparin with low dose aspirin in treatment of thrombo prophylaxis in utero placental insufficiency: a new vision in heparinization during pregnancy
}

\author{
M. Madhubala ${ }^{1}$, C. Kasthuri ${ }^{1}$, Mansi Shukul ${ }^{1}$, J. Mohamed Ali ${ }^{2}$
}

\author{
${ }^{1}$ Department of Obstetrics and Gynecology, Lakshmi Madhavan Hospital Obstetrics and Fertility Care Centre, \\ Tirunelveli, Tamil Nadu, India \\ ${ }^{2}$ Department of Clinical Research, Dr. Agarwal's Healthcare Limited, Tamil Nadu, India
}

Received: 11 November 2018

Accepted: 21 November 2018

\section{*Correspondence:}

Dr. C. Kasthuri,

E-mail: kasthu_mbbs@yahoo.co.in

Copyright: () the author(s), publisher and licensee Medip Academy. This is an open-access article distributed under the terms of the Creative Commons Attribution Non-Commercial License, which permits unrestricted non-commercial use, distribution, and reproduction in any medium, provided the original work is properly cited.

\section{ABSTRACT}

Background: The use of heparin and aspirin in obstetric care has grown considerably since their introduction into clinical practice. Because of the physiological changes of pregnancy, the usage of heparin and optimal dosage of heparin remains uncertain. Here our institute designed low dose Unfractionated Heparin (5000 IU s/c daily) as thrombo Prophylaxis regimen. To study the outcome of low dose UFH (5000 IU sc /daily) + Low dose aspirin (75 mg oral per day) for thrombo prophylaxis in utero placental insufficiency, in patient with 2 or more abortions.

Methods: This retrospective study was conducted in 135 patients with 2 or more abortions as obstetric history. Prophylactic low dose of UFH (5000 IU s/c daily) + LDA $75 \mathrm{mg}$ oral was initiated. The Primary outcome is live birth, and secondary outcomes is Reduced incidence of early onset of gestational hypertension (HT), Intrauterine Growth Retardation (IUGR).

Results: Out of 135 women 131 gave live birth, 2 had first trimester abortion and 2 had intrauterine death by 5 to 6 months. PIH was higher in patients with more than 30 years of age.

Conclusions: In our Retrospective, data combination of low dose UFH (5000IU s/c) + LDA (75mg oral) is as safe as routine thrombo prophylaxis with good compliance.

Keywords: Aspirin, Heparin, IUGR (Intrauterine Growth Retardation), Heparin, LMWH, LDA (Low dose Aspirin), Pregnancy induced hypertension (PIH), Unfractionated Heparin (UFH)

\section{INTRODUCTION}

Bad Obstetric History ( $\mathrm{BOH}$ ) is associated with adverse pregnancy outcomes, including recurrent miscarriage, fetal demise, maternal - Fetal complications such as preterm deliveries, Gestational Hypertension (HT), IUGR, Antepartum Hemorrhagic (APH), Abnormal fetal Doppler. Heparin exerts its primary anti-coagulant activity by binding to plasma Antithrombin (AT)-III. Neutralize the thrombin and prevent the extension of clot formation. As thrombin production diminishes the heparin dose needed may decrease. ${ }^{1}$ Recent studies suggested that therapeutic dose of UFH enhanced the differentiation of extra villous trophoplast into giant multinuclear cells, and so helps to correct the "defective endovascular trophoblastic invasion rather excessive inter villous thrombosis prevention as thought earlier. ${ }^{2}$

In $\mathrm{BOH}$ without any treatment only 5-10\% of pregnancies will be successful. ${ }^{3}$ Once daily regimen LMWH is acceptable for treatment of venous thrombo embolism (VTE) and it is licensed for non-pregnant 
individuals according to recent United Kingdom prospective surveys of the management of VTE. ${ }^{4}$

Could single dose be administered in pregnant women to prevent thrombo embolism in $\mathrm{BOH}$ patient? A study assessing Tinzaparin vs UFH in prophylactic treatment of VTE in patients undergoing general surgery, coupled with smith et al.'s results of tinzaparin use during pregnancy to prevent thrombosis in clinical trials was also available. ${ }^{5}$ So with all these references and in our semi urban setup we started on once daily - low dose $5000 \mathrm{IU}$ UFH + LDA $75 \mathrm{mg}$ oral as thrombo Prophylaxis, in Utero Placental Insufficiency, that is in patient with 2 or more abortions as obstetric history.

For many years UFH was standard anticoagulant used in pregnancy. LMWH have many advantages over UFH, but very costly for all patient to follow throughout the pregnancy with dose adjustment in second and third trimester. Low dose UFH, is associated with very lower risk of bleeding, reduced early onset of gestational HT, no incidence of Osteoporosis, no incidence of HIT, reduced risk of skin allergy and Predictable Pharmacokinetics without the need for frequent monitoring and very good patient compliance in our clinical practice.

And as many prescribed UFH + LDA reduced the complication of maternal / fetal outcome, we started on UFH and LDA as a routine protocol for all patient in our outpatient department, therefore designed a retrospective data of (Low dose UFH + LDA (75 mg) in 135 patients, with H/O utero placental insufficiency (2 or more abortions). ${ }^{6-9}$ To study the outcome of low dose UFH (5000 IU SC /daily) + Low dose aspirin (75 mg oral per day) for thrombo prophylaxis in utero placental insufficiency, in patient with 2 or more abortions.

\section{METHODS}

This retrospective study was conducted in a tertiary care obstetrics and gynecology hospital at Tirunelveli, South Tamil Nadu. Date was collected from 2015 December to 2018 June, cases with H/O recurrent pregnancy loss, unexplained fetal demise were enrolled in this study. Of 135 women with $\mathrm{H} / \mathrm{O}$ utero placental insufficiency, two groups were made. Group 1 - 2 abortions (111 women, with more than 10 weeks abortions + fetal demise). Group $2 \geq 3$ abortion ( 20 women).

As we are going with uterus - placental insufficiency as past history, no laboratory criteria were included in the study. Though we have clinical criteria to match as APS / $\mathrm{BOH}$, the broad terminology of utero - placental insufficiency is used, as of our clinical practice is considered.

All patients were trained to use s/c Inj. Heparin $5 \mathrm{ml}$ (25000 IU) vial. Each $\mathrm{ml}$ contains 5000IU. All were trained to give s/c injection, using Insulin syringe, since insulin syringe have short needle which is painless and easy to get trained. They were instructed to self administer 5000IU daily in medial side of thigh. Those patients who have developed discoloration of skin were advised to self - administer in lower abdomen, since there will be loose s/c fat.

All 135 women were started on low dose UFH + LDA (75 mg daily) as soon as Urine Pregnancy Test (UPT) is positive - before 6 weeks. All were done with transvaginal Sonography (TVS) @ 7-8 weeks to confirm cardiac activity. We continued LDA $75 \mathrm{mg}$, daily throughout the pregnancy up to 37 completed weeks. Once daily UFH - 5000IU (low dose) s/c given

- Daily up to 13-16 weeks.

- Alternative Days (from 15-17 weeks) to 22-24 weeks.

- Weekly twice 6th month- $7^{\text {th }}$ month.

- $\quad$ Biweekly ( $8^{\text {th }}$ to $9^{\text {th }}$ month).

- $\quad$ Stopped on 36 to 37 weeks.

All patient were serially scanned every visit, monthly once up to 7 th month, biweekly $7^{\text {th }}-9^{\text {th }}$ month, weekly after 9th month to see features suggestive of oligohydramnios, IUGR, Abnormal Doppler studies, and dosage of heparin will be continued in daily basic if any complication noted in serial ultrasonogram (USG).

Patients who becomes gestational HT and going for complications such as oligo, IUGR, Doppler abnormalities were changed to therapeutic protocol (LMWH 5000 IU daily s/c) and considered as failed low dose UFH. Those who don't go for complication are continued with once daily low dose UFH. Coagulation profile was repeated only for PIH women @ every 15 days, Prothrombin Time (PT), Activated Partial Thromboplastin Time (APTT), INR, Platelets.

\section{RESULTS}

Out of 135 women, enrolled in our retrospective study, Age $<29=49$ women, age $>30=86$ women, Married Duration out of 135 women $110=5.8$ years, 25 women $=$ 7.52 years. No of Abortions 2 abortions $=113, \geq 3$ abortions $=22$. Out of 135 women 131 gave live birth. 2 had first trimester abortion, 2 had intrauterine death by 5 to 6 months, 4 women had severe PIH (Table 1).

Table 1: Cross tabulation of number of incidences of abortion with final outcome.

\begin{tabular}{|c|c|c|c|c|}
\hline \multirow{2}{*}{ Group } & \multicolumn{2}{|l|}{ Deliverv } & \multirow{2}{*}{ Total } & \multirow{2}{*}{$\begin{array}{l}P \\
\text { value }\end{array}$} \\
\hline & SVD $(\%)$ & $\operatorname{LSCS}(\%)$ & & \\
\hline tions & $33 \quad 30.8$ & 69.2 & 107 & 0056 \\
\hline$\geq 3$ abortions & 10.0 & 18 & 20 & \\
\hline
\end{tabular}

Our primary outcome giving birth is $94 \%$ in 135 cases. Out of 131 women 24 become mild PIH (gestational HT) $18.5 \%$. Out of which 4 developed complications due to 
PIH. 20 mild PIH were continued with once daily - low dose UFH on daily basis. 4 with complicated - HT were shifted to single dose LMWH (5000 IU) and considered as failed low dose protocol.

18 cases in 2 abortions group had PIH, whereas 3 cases in more than 2 abortions group.

No significance between no of abortions and incidence of PIH. Failed once daily low dose have occurred in 2 abortions group (Table 2).

Table: 2 Cross tabulation of number of incidences of abortion with maternal complication.

\begin{tabular}{|l|lllll|}
\hline Group & PIH & \multicolumn{3}{c|}{ P } \\
\hline 2 abortions & 18 & $16.8 \%$ & 89 & $83.2 \%$ & \\
\hline 3 abortions & 3 & $15.0 \%$ & 17 & $85.0 \%$ & 0.84 \\
\hline
\end{tabular}

17 cases in 2 abortions group had baby weight less than $2.5 \mathrm{kgs}$, whereas 4 cases in more than 2 abortions group. No significance between no of abortions and fetal IUGR / Low birth weight (Table 3).

Table: 3 Cross tabulation of number of incidences of abortion with baby birth weight.

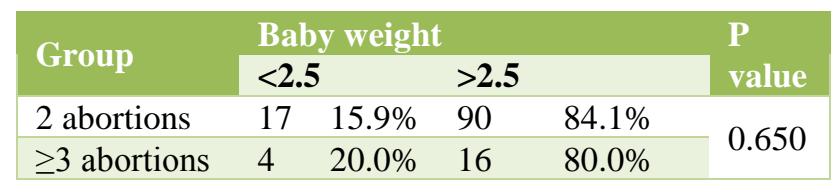

$23.5 \%$ cases in more than 30 years age group had incidence of PIH where as $8.7 \%$ cases in less than 30 years group. $\mathrm{P}$ value is Significant with age and incidence of PIH, incidence of gestational HT was found to be late onset (Table 4).

Table: 4 Cross tabulation of age group of women with maternal complication.

\begin{tabular}{|c|c|c|c|c|c|}
\hline \multirow{2}{*}{ Age group } & \multicolumn{4}{|c|}{ PIH } & \multirow{2}{*}{$P$ value } \\
\hline & Yes & & No & & \\
\hline$<=29$ & 4 & $8.7 \%$ & 43 & $93.5 \%$ & \multirow{2}{*}{0.042} \\
\hline$>30$ & 19 & $23.5 \%$ & 65 & $80.2 \%$ & \\
\hline
\end{tabular}

Table 5 shows the mean age of PIH incidence of present study is 30.83 years.

Table 5: Distribution of age in maternal complication.

\begin{tabular}{|lllll|}
\hline PIH & N & Mean & Std. Deviation & P value \\
\hline Yes & 23 & 30.83 & 4.41 & \multirow{2}{*}{0.004} \\
\hline No & 108 & 28.06 & 3.97 & \\
\hline
\end{tabular}

Marital duration of PIH patients is 7.24 \pm 4.2 years whereas $5.83 \pm 3.03$ years in other group. No significance between marital duration and incidence of PIH (Table 6).
Table 6: Distribution of marital duration in maternal complication.

\begin{tabular}{llllll} 
PIH & & N & Mean & Std. Deviation & P value \\
Marital & Yes & 23 & 7.24 & 4.2 & \multirow{2}{*}{0.062} \\
\cline { 2 - 6 } duration & No & 108 & 5.83 & 3.03 & \\
\hline
\end{tabular}

\section{DISCUSSION}

Early pregnancy loss or miscarriage overall is estimated to occur in $12-15 \%$ of clinically recognized pregnancies (Regan and Rai; Rai and Regan). ${ }^{10,11}$ Recurrent loss, defined as three or more consecutive losses, occurs in $1 \%$ of women (Regan and Rai, Rai and Regan). ${ }^{10,11}$ This prevalence estimate increases to $3-5 \%$ if defined as two or more losses (Regan and Rai; Rai and Regan). ${ }^{10,11}$ Various interventions have been considered to improve the subsequent live birth rates for women with recurrent pregnancy loss. Consideration of a thrombotic origin for recurrent loss came from initial observational studies showing associations with both inherited and acquired (APS) thrombophilia (Rai and Regan). ${ }^{11}$

Our results suggest that low dose - UFH + LDA (75 mg) for thrombo prophylaxis of utero placental insufficiency is as effective as management options discussed in present study. Although limited data for criteria of APS are available (only clinical criteria) we have determined that our protocol is effective. Efficacy, safety, cost effective and good compliance of patient is well met with our clinical outcome. Primary Outcome of giving birth is $94 \%$ with low dose UFH 5000IU s/c in 135 cases. Secondary outcome is there is a reduction in incidence of PIH. All were found to be late onset gestational HT. Gestational HT with complications are considered as "failed in low dose" and changed over to therapeutics dose, LMWH. Out of 135 women 4 had no live birth 4 were considered as failed low dose UFH. So out of 127 women 124 had full term deliveries, 3 preterm (32-35 weeks) deliveries, 20 had mild PIH, 3 IUGR babies (1.5 to $1.7 \mathrm{~kg}), 1 \mathrm{APH}$. No excessive bleeding during delivery, No DVT, No HIT, no eclampsia / Preeclampsia, bone fractures, 10 women had allergic rashes. Although bone density was not assessed in present study $1 \mathrm{gm}$ of calcium daily is given as oral supplement. UFH was stopped at 36 weeks- 37 weeks and LDA stopped at 37 weeks. It is well known that Heparin and aspirin reduced the pregnancy loss, decreased the onset of gestational HT, decreased the complications (maternal / fetal outcome during pregnancy). The strength of the study is it is purely a clinical based study. Lower prophylactic dosage, No increase in dosage after 20 weeks, stable pharmacokinetics and coagulation profile, with reduced risk of Heparinization. Although the smaller sample size reduces the precision of the findings, we still are able to confirm our hypothesis that the unfractionated heparin regimen is not inferior to low-molecular-weight heparin. Thus, reduced considerable financial and emotional load associated with such adverse outcome. Considering each pregnancy as very precious a broad terminology of utero 
placental insufficiency is used to reduce the hesitance to start on heparin and not to wait for greater than 3 consecutive abortions (as per guidelines). Though many trials like FRUIT trial are there as a country like India, with poor resources for health benefit for low socioeconomic status, this low lost as effective as good compliance protocol will suffice for prophylactic measures. ${ }^{8,12-16}$

\section{CONCLUSION}

(Low dose UFH + LDA) is as effective as routine thrombo prophylaxis as per old guidelines, with good maternal and fetal outcome, without affecting the patient's economy and compliance. LMWH may be effective in certain patients but most of the patients will have good obstetric outcome with our low dose UFH protocol. Large group studies are required.

Funding: No funding sources

Conflict of interest: None declared

Ethical approval: The study was approved by the Institutional Ethics Committee

\section{REFERENCES}

1. Heuser, C., and Ware Branch, D. (n.d.). Disorders of Coagulation in Pregnancy. In D. James, P. Steer, C. Weiner, B. Gonik, and S. Robson (Eds.), Cambridge: Cambridge University Press. High-Risk Pregnancy: Management Options pp. 1085-1107.

2. Noble LS, Kutteh WH, Lashley N, Franklin RD, Herrada J. Antiphospholipid antibodies (APA) associated with recurrent pregnancy loss: prospective, multicenter, controlled pilot study comparing treatment with enoxaparin versus unfractionated heparin. Fertil Steril.2005;83(3):68490.

3. Stephenson M, Ballem P, Tsang P, Purkiss S, Ensworth S, Houlihan E et al. Treatment of Antiphospholipid Antibody Syndrome (APS) in Pregnancy: A Randomized Pilot Trial Comparing Low Molecular Weight Heparin to Unfractionated Heparin. J Obstet Gynaecol Canada. 2004;26(8):72934.

4. Patel JP, Hunt BJ. Where do we go now with low molecular weight heparin use in obstetric care? J Thromb Haemost. 2008;6(9):1461-7.

5. Smith MP, Norris LA, Steer PJ, Savidge GF, Bonnar J. Tinzaparin sodium for thrombosis treatment and prevention during pregnancy. Am J Obstet Gynecol. 2004;190(2):495-501.

6. IanA.Greer and Catherine Nelson Piercy. Low Molecular Weight Heparin FOR Thrombo Prophylaxis and Treatment of Venous Thrombo Embolism in Pregnancy. Blood. 2005;106(2):401-7.

7. James D, Steer P, Weiner C Gonik B. High risk pregnancy. Philadelphia, PA: $4^{\text {th }}$ Saunders/Elsevier; 2011.

8. Queenan J, Spong C, Lockwood C. Queenan's management of high-risk pregnancy. Chichester, West Sussex: Wiley-Blackwell; 2012.

9. Williams J. Obstetrics. Stamford, Conn.: Appleton and Lange; 1997.

10. Regan L, Rai R. Epidemiology and the medical causes of miscarriage. Best practice and research Clinic Obstet Gynaecol. 2000;14(5):839-54.

11. Rai R, Regan L. Recurrent miscarriage. The Lancet. 2006;368(9535):601-11.

12. van Hoorn ME, Hague WM, van Pampus MG, Bezemer D, de Vries JI. Low-molecular-weight heparin and aspirin in the prevention of recurrent early-onset pre-eclampsia in women with antiphospholipid antibodies: the FRUIT-RCT. European J Obstet Gynecol Reproduct Biol. 2016;197:168-73.

13. Dresang LT, Fontaine P, Leeman L, King VJ. Venous thromboembolism during pregnancy. Am Fam Physician. 2008;77(12).

14. Steinberg ZL, Dominguez-Islas CP, Otto CM, Stout KK, Krieger EV. Maternal and Fetal Outcomes of Anticoagulation in Pregnant Women with Mechanical Heart Valves. J Am Coll Cardiol. 2017;69(22):2681-91.

15. Salmon J, Girardi G, Lockshin M. The antiphospholipid syndrome as a disorder initiated by inflammation: implications for the therapy of pregnant patients. Nature Clinical Practice Rheumatol. 2007;3(3):140-7.

16. Backos M, Rai R, Thomas E, Murphy M, Doré C, Regan L. Bone density changes in pregnant women treated with heparin: a prospective, longitudinal study. Human Reproduct. 1999;14(11):2876-80.

Cite this article as: Madhubala M, Kasthuri C, Shukul M, Ali JM. Low dose unfractionated heparin with low dose aspirin in treatment of thrombo prophylaxis in utero placental insufficiency: a new vision in heparinization during pregnancy. Int $\mathbf{J}$ Reprod Contracept Obstet Gynecol 2018;7:4849-52. 